\title{
High-dose-rate fractionated brachytherapy monotherapy for localized prostate cancer: a systematic review and meta-analysis
}

\author{
Eric M. Anderson, MD',2, Sungjin Kim, MS 2,3, Howard M. Sandler, MD',2, Mitchell Kamrava, MD',2 \\ 'Department of Radiation Oncology, Cedars-Sinai Medical Center, Los Angeles, CA, USA, ${ }^{2}$ Samuel Oschin Comprehensive Cancer Institute, \\ Cedars-Sinai Medical Center, Los Angeles, CA, USA, ${ }^{3}$ Biostatistics and Bioinformatics Research Center, Cedars-Sinai Medical Center, \\ Los Angeles, CA, USA
}

\begin{abstract}
Purpose: High-dose-rate (HDR) brachytherapy as primary therapy (monotherapy) is a standard National Comprehensive Cancer Network (NCCN) endorsed treatment option for patients with localized prostate cancer. Thus far, most data are limited to single-institution experiences. Accordingly, we sought to systematically review rates of biochemical recurrence-free survival (bRFS) and toxicity associated with fractionated HDR monotherapy.

Material and methods: A systematic review was performed using PubMed and Embase databases for relevant articles published between January 1999 and December 2019, according to preferred reporting items for systematic review and meta-analyses (PRISMA) guidelines. Included studies were limited to fractionated HDR monotherapy publications in full manuscript form with at least 5-year median follow-up, at least 80 patients included, and adequate reporting of bRFS and toxicity data. Meta-analyses were performed with random-effect modeling. Extent of heterogeneity between studies was determined using $I^{2}$ and Cochran's $Q$ tests.

Results: Seven unique studies were identified, including 2,123 patients. NCCN low-, intermediate-, and high-risk patients comprised $40 \%, 40 \%$, and $20 \%$ of patients, respectively. Median follow-up at the study group level was 74 months (range, 60-131 months). The 5-year bRFS rate was 95\% (95\% confidence interval [CI]: 93-96\%), and after adjusting to control for publication bias, it was $96 \%$ (95\% CI: 94-99\%). Estimated adjusted late grade $\geq 3$ genitourinary and gastrointestinal toxicity rates were $2 \%$ (95\% CI: 1-4\%) and $0.3 \%$ (95\% CI: 0-1.1\%), respectively.

Conclusions: Fractionated HDR monotherapy is associated with high rates of disease control and low rates of toxicity. Future studies are needed to better define the value of this treatment modality relative to other options.

Key words: high-dose-rate brachytherapy, prostate cancer, biochemical recurrence-free survival, meta-analysis.

\section{Purpose}

Brachytherapy as monotherapy is supported by National Comprehensive Cancer Network (NCCN) guidelines for low- and favorable intermediate-risk patients. It can be performed with either low-dose-rate (LDR) or high-dose-rate (HDR) brachytherapy. The National Cancer Database (NCDB) analysis suggests that LDR is performed more frequently than HDR monotherapy [1]. There are no major prospective randomized trials comparing LDR with HDR to guide whether one should be performed over another, but small studies suggest possible improvement of urinary quality of life with HDR [2].

Until randomized data becomes available, efforts to better understand the efficacy and toxicity of each modality are important. Given that HDR is a newer modality compared with LDR, there is a smaller overall body of published literature. Most data come from single-institution series, and there are limited prospective studies with median follow-up greater than five years. Consequently, we performed a systematic review and meta-analysis of fractionated HDR monotherapy series with greater than 5 years median follow-up in an effort to better understand disease control rates and toxicity outcomes with this technique. While a recent meta-analysis on HDR monotherapy was published, it included single-fraction HDR monotherapy, which is known to have inferior outcomes compared with multi-fraction regimen [3]. On a meta-regression analysis in this prior study, the number of fractions was associated with biochemical failure, but a separate analysis of only patients treated with 
multi-fractionated HDR monotherapy regimens was not performed. Assuming that multi-fractionated HDR monotherapy is the current standard of care, we focused our systematic review and meta-analysis on patients treated with multi-fractionated HDR monotherapy only.

\section{Material and methods}

\section{Search strategy and study selection}

This systematic review and meta-analysis was performed in accordance with the preferred reporting items for systematic review and meta-analyses (PRISMA) guidelines [4]. We searched PubMed and Embase databases for published English language titles between January 1, 1999 and December 31, 2019. Search terms included variants of "prostate" and "cancer" and "brachytherapy or radiation or radiotherapy". A full list of search criteria is presented in Supplement 1. We included only published original research manuscripts, and excluded conference proceedings, task force reports, professional organization statements, and conference abstracts.

Only studies reporting findings on patients receiving fractionated HDR brachytherapy as monotherapy, treated in definitive setting for prostate adenocarcinoma, with apparent clinical outcome and toxicity data, were included. Studies reporting findings on patients who received single-fraction HDR monotherapy were excluded given reported inferior outcomes, which was not considered a standard approach [3]. In addition, only studies including at least 80 patients with median follow-up greater than or equal to 5 years were included. Studies were excluded if patients received HDR for salvage therapy, had prior pelvic radiation treatment, or were noted to have a unique aspect to the cohort, such as patients having inflammatory bowel disorder. A prospective study that was published beyond the cutoff date for inclusion in the formal database search was also included in the analysis [3]. The PRISMA flow chart with records from each step were independently reviewed by M.K. and E.A. (Supplemental Figure 1).

A total of 916 and 522 records were identified in the Embase and PubMed databases, respectively. Among these 1,438 records, 910 were found to be duplicates, yielding 521 unique records. Of 521 records screened, 36 review articles, letters to the editor, and editorials were excluded. The remaining 485 apparent original journal articles were assessed for eligibility by M.K. and E.A., and 420 were excluded based upon treatment and patients' characteristics not meeting our criteria for inclusion. Additionally, we excluded 51 studies reporting less than 5-year median follow-up, and/or studies apparently reporting on the same cohort of patients. If cohorts appeared to have been previously reported, only the most recent publication was included in this analysis. For example, a single study by Demanes et al. [5] was excluded, as it was felt that the patients from this study were likely included (at least in part) in a subsequent report by Hauswald et al. [6]. Two other studies, Yoshida et al. [7] and Yoshioka et al. [8] also appeared to overlap with the report by Yamazaki et al. [9], with less well-described outcomes' data, and these two studies were accordingly excluded. This left a total of seven studies for further analysis.

\section{Data extraction}

Two authors (M.K. and E.A.) independently extracted data from the seven studies [3, 6, 9-13]. Variables extracted included sample size, dose per fraction, fraction number, median follow-up, median patient age, clinical tumor stage, median pre-treatment prostate-specific antigen (PSA), Gleason score, NCCN risk group, receipt of androgen-deprivation therapy (ADT), biochemical recurrence-free survival (bRFS), and physician-reported toxicity. Individual patients' data were not used. Biochemical failure (BF) was defined according to Phoenix consensus definition [14] in all included studies. bRFS was defined by BF events, only in 5 of 7 included studies. In the other two studies, bRFS was a combined endpoint, with both $\mathrm{BF}$ and initiation of salvage hormonal therapy. None of the included studies specified whether death was censored or considered an event.

\section{Statistical analysis}

Primary outcomes comprised 5-year bRFS, physician-reported late grade $\geq 3$ genitourinary (GU), and gastrointestinal (GI) toxicity rates. Toxicity grading was in accordance with physician's report, and corresponded to common terminology criteria for adverse events (CT$\mathrm{CAE}$ ) version reported per individual publication. As we considered multiple independent study groups from the same publication, a multi-level structure of data (study group as level-2 unit and publication as level-3 unit) was examined by adding two random effects at both the second- and third-level to the model with a three-level structure [15]. Since the third-level (publication) variance and intra-class correlation coefficient (ICC, correlation between study groups within publications) were insignificantly small [15], meta-analyses were performed using a standard random-effects model with a two-level structure to estimate pooled outcome rates, given the extent of heterogeneity across study groups assessed with Cochran's Q test and $I^{2}$ value [16]. Study weight was estimated based on the inverse of the variance. Meta-analyses were also carried out using a random-effects model to estimate pooled bRFS rates at 4-, 5-, 6-, and 7-years status post-HDR brachytherapy treatment. Meta-regression was further conducted to examine whether 5-year bRFS rate, and late grade $\geq 3 \mathrm{GU}$ and GI toxicity rates were associated with biologically equivalent dose (BED) and NCCN risk group. Of note, data from Hauswald et al. included in meta-analyses were for 6-year bRFS rather than 5-year bRFS, as was reported for all other studies. It was acknowledged that inclusion of this 6-year bRFS data point from Hauswald et al. may lead to an overestimation of failure rates in this study compared to other studies included in meta-analysis, which reported 5-year bRFS. BED was calculated assuming an $\alpha / \beta$ ratio of $1.5\left(B_{1.5}\right)$. Publication bias was examined using funnel plots and Egger's test [17]. Adjusted outcome rates were estimated 
using Duval and Tweedie's trim-and-fill method to control publication bias [18]. All analyses were performed using $\mathrm{R}$ package version 3.5.3 with two-sided tests, at a significant level of 0.05 .

\section{Results}

A total of 2,123 patients were included from the seven studies considered for analysis. Additionally, three studies (Hoskin et al., Yamazaki et al., and Zamboglou et al.) contained various dose/fraction schemes, and each of these was treated as a separate group, thereby making a total of 11 study groups. Patients' baseline demographic, treatment, and tumor characteristics are presented in Table 1 . The median age of a patient at diagnosis was 66 years (range, $62-71$ years). Most patients presented with clinical T1 $(52 \%)$ or T2 $(39 \%)$ disease. The median PSA was reported in 7 of 11 study groups, corresponding to an overall median PSA of $7.5 \mathrm{ng} / \mathrm{ml}$ (range, $6-17.4 \mathrm{ng} / \mathrm{ml}$ ) across study groups. Most patients had either Gleason 6 or lower $(56 \%)$ or Gleason $7(38 \%)$ disease. Although most patients had NCCN low- $(40 \%)$ or intermediate$(40 \%)$ risk disease, $20 \%$ of patients had high-risk disease. A total of 671 patients $(32 \%)$ received ADT as a part of their initial definitive treatment course. The median ADT duration was reported in 9 of 11 study groups, and was at least 7 months in 5 of 9 study groups reported. The median number of radiation treatment fractions was 5 (range, 2-9 fractions), and the median dose per fraction was 8.8 Gy (range, 6-13.5 Gy). The median follow-up at the study group level was 74 months (range, 60-131 months).

The pooled estimate of 5-year bRFS rate was $0.95 \%$ (95\% CI: $0.93-0.97 \%, p$-value < 0.001) using random-effects model (Figure 1). Estimated effects against corresponding standard errors evaluated by Egger's test indicated publication bias ( $p$-value $<0.001$ ), and the adjusted pooled 5-year bRFS rate after controlling for publication bias was $0.96 \%$ (95\% CI: 0.94-0.99\%, $p$-value < 0.001) (Supplemental Figure 2). Additionally, study groups with higher percentages of patients with high-risk disease were generally found to have higher rates of biochemical failure (Figure 1). Meta-regression confirmed this finding by revealing that an increasing percentage of patients with high-risk disease within each study cohort was associated with decreasing rates of 5 -year bRFS ( $p$-value $=$ 0.004) (Supplemental Figure 3). Meta-analysis of bRFS reported at 4-, 5-, 6-, and 7-years follow-up across study groups revealed an expected downward trend of bRFS with increasing follow-up duration (Figure 2).

Radiation treatment dose and fractionation varied significantly across study groups. The total number of patients, dose per fraction in Gy, number of fractions, and $\mathrm{BED}_{1.5}$ reported by study group are presented in Table 2 . Of note, for the Zamboglou et al. study, the difference between study cohorts was the timing of radiation treatment as patients in group 1 were treated twice daily over two days using a single-implant, while patients in group 2 were treated with two implants, two weeks apart, and two fractions delivered over 12 hours with each implant. $\mathrm{BED}_{1.5}$ ranged between 243 and $279 \mathrm{~Gy}$ across the studies,
Table 1. Patients' baseline demographics, treatment, and tumor characteristics. Patients' level data were reported with the total number across studies and corresponding percentages. Mean values across studies were weighted by the number of patients per trial and reported with corresponding ranges. Median prostate specific antigen (PSA) values were available in seven study groups reported. Median duration of androgen deprivation therapy (ADT) treatment was available in nine study groups reported

\begin{tabular}{lc} 
Variable & $\begin{array}{c}\text { Patient level } \\
(\%)\end{array}$ \\
\hline $\begin{array}{l}\text { Total number }{ }^{*} \text { (range) } \\
\text { the studies }\end{array}$ & 2,123 \\
\hline Number of fractions & $5(2,9)$ \\
\hline Dose per fraction (Gy) & $8.8(6,13.5)$ \\
\hline Median age (years) & $66(62,71)$ \\
\hline Clinical stage & $1,109(52.2)$ \\
\hline T1 & $822(38.7)$ \\
\hline T2 & $188(8.9)$ \\
\hline T3/T4 & $4(0.2)$ \\
\hline Unknown & $7.5(6.3,17.4)$ \\
\hline Median PSA (ng/ml) ${ }^{1}$ & \\
\hline Gleason score & $1,197(56.4)$ \\
\hline$\leq 6$ & $742(38.0)$ \\
\hline 7 & $120(5.6)$ \\
\hline$\geq 8$ & $871(31.6)$ \\
\hline NCCN risk group & $1,503(81.2)$ \\
\hline Low & $860(40.5)$ \\
\hline Intermediate & $420(19.8)$ \\
\hline High & \\
\hline ADT receipt & \\
\hline Median ADT duration $<7$ months $^{2}$ & \\
\hline
\end{tabular}

*Mean values are weighted by the number of patients per trial, ${ }^{1}$ seven study groups reported, ${ }^{2}$ nine study groups reported, Gy - Gray, n-sample size, NCCN - National Comprehensive Cancer Network

with the vast majority of patients receiving between 250$280 \mathrm{~Gy} \mathrm{BED}_{1.5}$. In meta-regression, 5-year bRFS was associated directly with $\mathrm{BED}_{1.5}(p$-value $=0.047)($ Figure 3$)$.

Figure 4 shows rates of late grade $\geq 3 \mathrm{GU}$ and GI toxicity with corresponding confidence intervals by study group as well as combined estimates using randomeffects modeling. The pooled estimate of late grade $\geq 3 \mathrm{GU}$ toxicity rate was $0.03 \%(95 \% \mathrm{CI}: 0.02-0.05 \%, p$-value $<0.001$ ) using a random-effect model. Estimated effects against corresponding standard errors evaluated by Egger's testing indicated publication bias ( $p$-value $<0.001$ ), and the adjusted pooled late grade $\geq 3$ GU toxicity rate after controlling for publication bias was $0.02 \%$ 


\begin{tabular}{|c|c|c|c|c|c|c|}
\hline \multirow[t]{2}{*}{ Study } & \multirow[t]{2}{*}{ Sample size } & \multicolumn{3}{|c|}{ NCCN risk group (\%) } & & \multirow[t]{2}{*}{5 -year bRFS rate $(95 \% \mathrm{CI})$} \\
\hline & & Low & Intermediate & High & & \\
\hline Hauswald et al. ${ }^{*}$ & 448 & 64 & 36 & 0 & $H$ & $0.99(0.98,1.00)$ \\
\hline Jawad et al. & 319 & 73 & 27 & 0 & $\mapsto-1$ & $0.97(0.95,0.99)$ \\
\hline Patel et al. & 190 & 0 & 100 & 0 & $\longmapsto$ & $0.97(0.95,0.99)$ \\
\hline Morton et al. & 83 & 14 & 86 & 0 & $\longmapsto$ & $0.95(0.90,1.00)$ \\
\hline Zamboglou et al. $(1)^{* *}$ & 141 & 73 & 16 & 11 & $\longmapsto-$ & $0.97(0.94,1.00)$ \\
\hline Zamboglou et al. (2)*** & 351 & 56 & 23 & 21 & $\longmapsto$ & $0.94(0.92,0.96)$ \\
\hline Yamazaki et al. (1) & 86 & 0 & 56 & 44 & $\longmapsto$ & $0.88(0.81,0.95)$ \\
\hline Yamazaki et al. (2) & 149 & 17 & 35 & 48 & $\longmapsto$ & $0.97(0.95,1.00)$ \\
\hline Yamazaki et al. (3) & 112 & 2 & 35 & 63 & - & $0.92(0.87,0.97)$ \\
\hline Hoskin et al. (1) & 138 & 0 & 50 & 50 & $\longmapsto$ & $0.90(0.85,0.95)$ \\
\hline Hoskin et al. (2) & 106 & 0 & 46 & 54 & $\longmapsto$ & $0.90(0.84,0.96)$ \\
\hline
\end{tabular}

*6-year bRFS, ** treated twice daily over 2 days using a single implant, ${ }^{* * *} 2$ implants, 2 weeks apart, and 2 fractions delivered over 12 hours with each implant

Overall

$\mathrm{Q}=22.96(p$-value $=0.006)$

$I^{2}=66.17 \%$

$0.95(0.93,0.97)$

$\begin{array}{lllll}0.80 & 0.85 & 0.90 & 0.95 & 1.00\end{array}$

Fig. 1. Forest plot for 5 -year biochemical recurrence-free survival (bRFS) rates with corresponding $95 \%$ confidence intervals in 11 individual study groups, and the combined estimate using a random-effects modeling. Study group patient sample size as well as percentage of patients within each NCCN risk group were also reported. The pooled estimate of 5-year bRFS rate was $0.95 \%(95 \%$ CI: $0.93-0.97 \%, p$-value $=0.0001)$. Of note, the data from Hauswald $e t$ al. included in this figure were for 6-year bRFS rather than 5-year bRFS, as was reported for all other studies. In the first study cohort published by Zomboglou et al., the patients were treated twice daily over 2 days using a single-implant, while patients in the second group were treated with 2 implants, two weeks apart, and two fractions delivered in a single-day each time (12 hours elapsed between twice daily treatments)

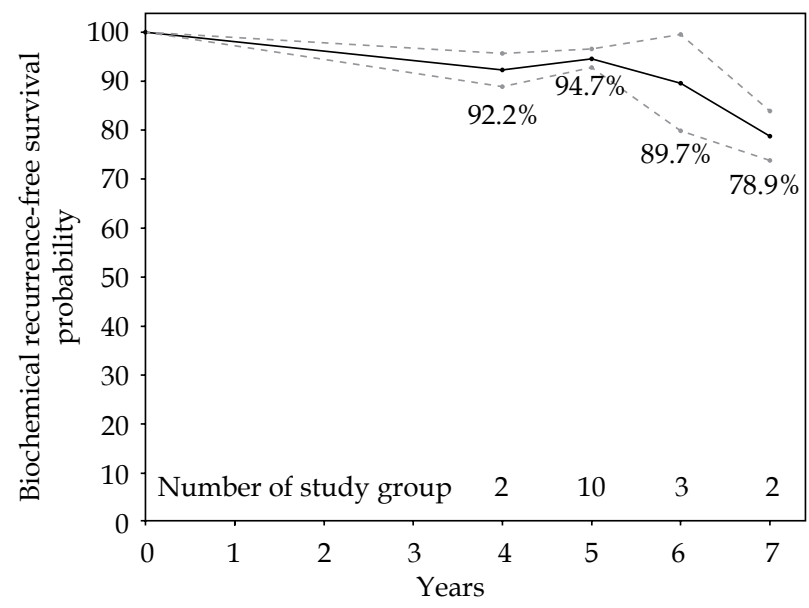

Fig. 2. Meta-analysis of biochemical recurrence-free survival (bRFS) rate in multiple studies at 4-, 5-, 6-, and 7 -years follow-up. Estimated values and corresponding 95\% confidence intervals are connected for illustrative purpose only
$(95 \%$ CI: $0.01-0.04 \%, p$-value $=0.008)($ Supplemental Figure 4). In meta-regression, late grade $\geq 3 \mathrm{GU}$ toxicity rates were not associated with biologically equivalent dose $(p$-value $=0.679)($ Supplemental Figure 6) .

The pooled estimate of late grade $\geq 3$ GI toxicity rate was $0.0002 \%(95 \%$ CI: $-0.001-0.001 \%, p$-value $=0.743)$ using a random-effect model (Figure 4). Estimated effects against corresponding standard errors evaluated by Egger's testing indicated publication bias ( $p$-value $=$ 0.005 ), and the adjusted pooled late grade $\geq 3$ GI toxicity rate after controlling for publication bias was $0.003 \%$ (95\% CI: $-0.006-0.011 \%, p$-value $=0.539)$ (Supplemental Figure 5). In meta-regression, late grade $\geq 3$ GI toxicity rates were not associated with BED $(p$-value $=0.687)$ (Supplemental Figure 7).

\section{Discussion}

This meta-analysis summarizes clinical outcomes and toxicities associated with definitive fractionated HDR monotherapy. The 5-year pooled estimate bRFS rate was 
Table 2. Total number of patients, dose per fraction in Gray (Gy), number of fractions, and biologically equivalent dose assuming $\alpha / \beta$ ratio of $1.5\left(B D_{1.5}\right)$ by study group. Study groups were defined by patients who received the same radiation dose and fractionation within a given study, and some studies therefore had multiple corresponding cohorts. Although all patients in the Zamboglou et al. study were treated with the same dose and fractionation, some patients were treated twice daily over two days using a single-implant, while others were treated with two implants, two weeks apart, and two fractions delivered over 12 hours with each implant

\begin{tabular}{lcccc}
\hline Study group & Number of patients & Dose per fraction (Gy) & Number of fractions & BED $_{1.5}(\mathrm{~Gy})$ \\
\hline Morton et al. & 83 & 13.5 & 2 & 270 \\
\hline Zamboglou et al. (1) ${ }^{*}$ & 141 & 9.5 & 4 & 279 \\
\hline Zamboglou et al. (2) ${ }^{*}$ & 351 & 9.5 & 4 & 279 \\
\hline Yamazaki et al. (1) & 86 & 6.5 & 7 & 243 \\
\hline Yamazaki et al. (2) & 149 & 7.0 & 9 & 278 \\
\hline Yamazaki et al. (3) & 112 & 6.0 & 6 & 270 \\
\hline Patel et al. & 190 & 7.25 & 4 & 254 \\
\hline Jawad et al. & 319 & 9.5 & 2 & 279 \\
\hline Hoskin et al. (1) & 138 & 13.0 & 3 & 252 \\
\hline Hoskin et al. (2) & 106 & 10.5 & 6 & 254 \\
\hline Hauswald et al. & 448 & 7.25 & 7 \\
\hline Patients were treated twise
\end{tabular}

* Patients were treated twice daily over two days using a single-implant, ** patients were treated with two implants, two weeks apart, and two fractions delivered over 12 hours with each implant

$95 \%$, adjusted to $96 \%$ after controlling for publication bias. The estimated 6- and 7-year bRFS rates were $89.7 \%$ and $78.9 \%$, respectively. These are encouraging results, particularly given that approximately $20 \%$ of the patients were NCCN high-risk patients. The fact that rates of bRFS decreased with an increasing proportion of high-risk patients is also consistent with the literature [19]. These bRFS rates are also similar to other standard treatments, including LDR [20], stereotactic body radiotherapy (SBRT) [19], and hypofractionated external beam radiation [21]. Additional follow-up time is needed to better understand whether clinically significant differences in bRFS between these modalities develop with time, especially that many prostate cancer-specific events occur between 5 to 10 years of follow-up.

We also found a relationship between bRFS and BED, which is consistent with a previously published metaanalysis on HDR monotherapy. Validation of this finding, when limiting the results to only multi-fractionated HDR monotherapy regimens, is helpful as multi-fractionated approaches are the current standard of care. While there is no standard fractionation scheme for HDR monotherapy, there is a general trend to decrease the total number of fractions, and the two regimens suggested by current NCCN guidelines are $13.5 \mathrm{~Gy} \times 2$ fractions or $9.5 \mathrm{~Gy} \times 4$ fractions, which correspond to BED of 270 and 279 , respectively. There are data demonstrating correlations between HDR boost in combination with external beam showing a higher chance of achieving a 5-year PSA $\leq 0.2 \mathrm{ng} / \mathrm{ml}$ with a total BED $>260$ Gy [22] and improvements in 10-year biochemical control with BED $>268$ [23]. Both of NCCN suggested HDR regimens have
BED dose levels above these thresholds seen for improved outcomes. They are also closer to the higher end of BED range among studies included in this meta-analysis (BED range, 240-280). We were unable to determine whether improvements in bRFS with increasing BED are limited to specific NCCN risk groups. This is an important unanswered question to be addressed in future studies.

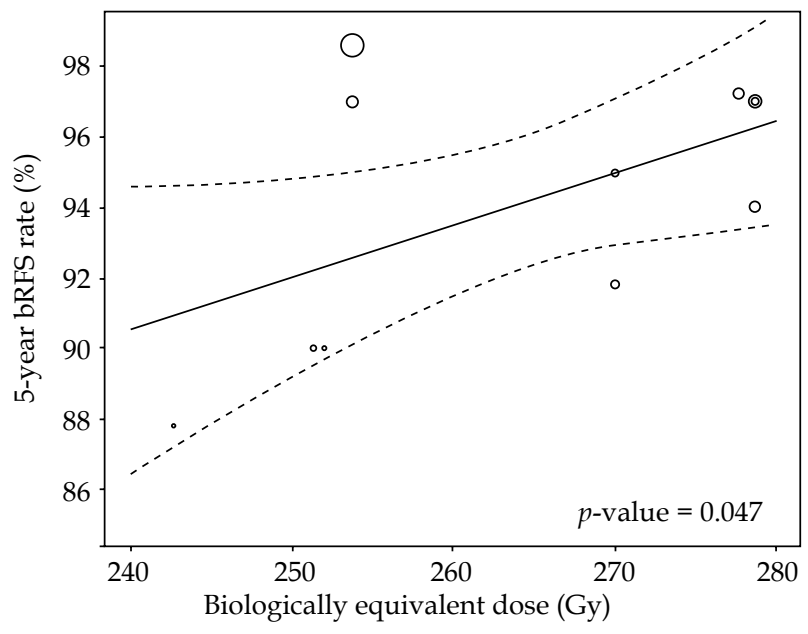

Fig. 3. 5-year biochemical recurrence-free survival (bRFS) rates with corresponding $95 \%$ confidence intervals. 5-year bRFS rates were associated directly, with biologically equivalent dose assuming $\alpha / \beta$ ratio of 1.5 in meta-regression $(p$-value $=0.047)$. Of note, the data from Hauswald et al. included in this figure were for 6-year bRFS rather than 5 -year bRFS, as was reported for all other studies 


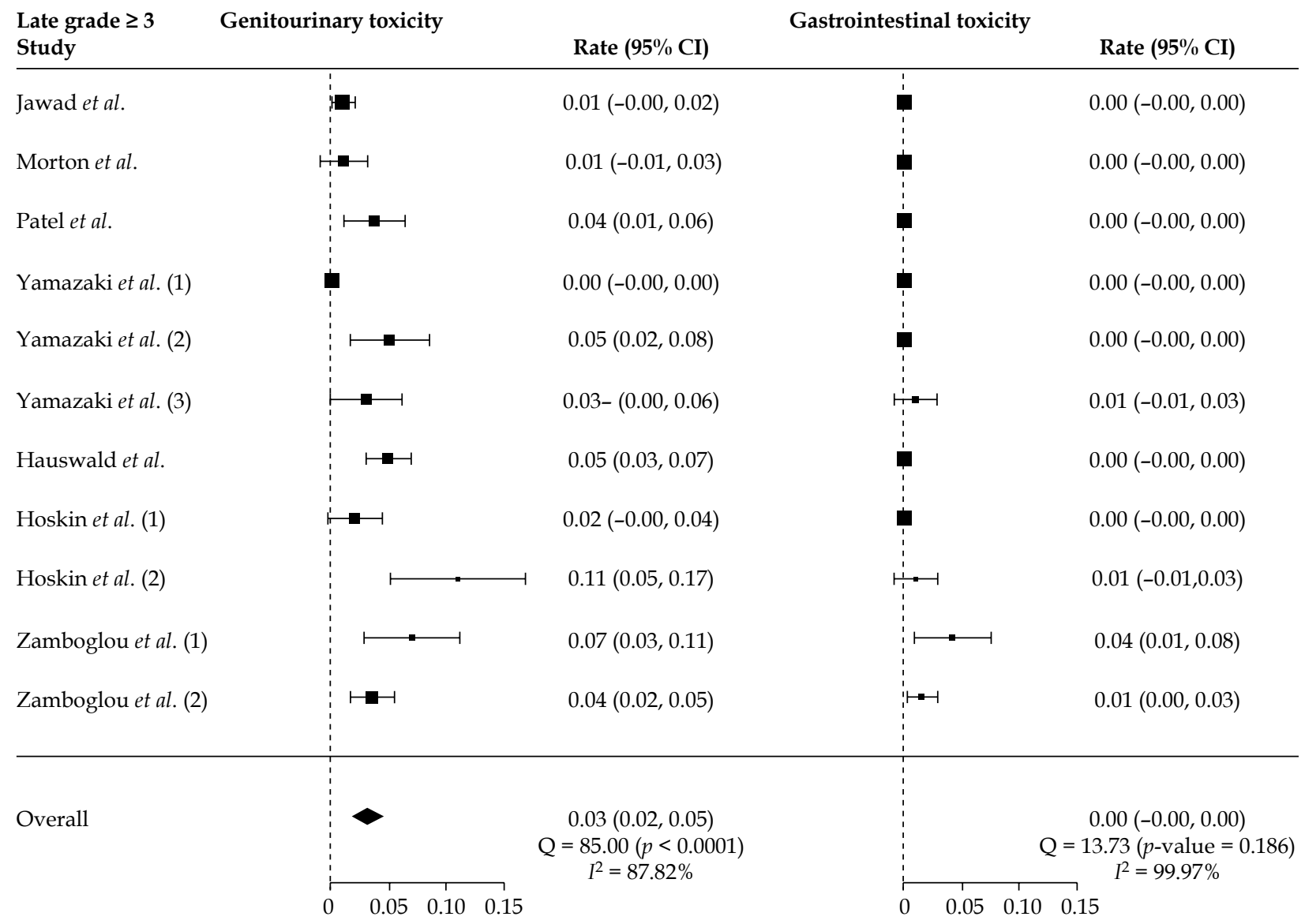

Fig. 4. Forest plots for late grade $\geq 3$ genitourinary (GU) and gastrointestinal (GI) toxicity rates with corresponding $95 \%$ confidence intervals in 11 individual study groups, and the combined estimate using a random-effects model. The pooled estimate of late grade $\geq 3 \mathrm{GU}$ toxicity rate was $0.03 \%$ ( $95 \% \mathrm{CI}: 0.02-0.05 \%, p$-value $<0.001)$. The pooled estimate of late grade $\geq 3 \mathrm{GI}$ toxicity rate was $0.0002 \%(95 \% \mathrm{CI}:-0.001-0.001 \%, p$-value $=0.743)$

Despite the relatively high BED delivered with HDR monotherapy, late $\geq$ G3 GU and GI toxicities were limited. The pooled late $\geq$ G3 GU toxicity rate across study groups was 2-3\%, and the pooled GI toxicity rate was less than $0.5 \%$. A statistically significant association was not observed between BED and rates of late $\geq$ G3, or higher GU or GI toxicities. These results are reassuring given the concerns of increased toxicities seen with LDR brachytherapy boost in the ASCENDE-RT trial [24].

This meta-analysis includes over 2,000 patients treated with fractionated HDR monotherapy. Individual studies, to date, have included a small number of patients, and prospective trials in HDR monotherapy have been limited to centers of brachytherapy excellence [3]. This poses a potential issue for inclusion of HDR monotherapy on national trials, with a concern for adequate expertise and subsequent feasibility of widespread adoption. This perception of difficulty with adoption of brachytherapy monotherapy is also reflected in the diminishing percentage of patients treated with either LDR or HDR monotherapy compared with the increasing number of patients being treated with SBRT $[1,25]$. This is all despite the fact that HDR and LDR monotherapy provide high-value compared with other standard external beam approaches [26].
A recent systematic review and meta-analysis was published and reported similar results, including 5-year bRFS $>90 \%$, late grade 3 or higher GU/GI toxicity rates of less than $2 \%$, and a correlation between BED and bRFS [27]. A limiting factor of this analysis is that it included patients' cohorts treated with a single-fraction HDR brachytherapy, which is associated with inferior outcomes and is therefore not considered as a standard of care [3]. This previous study also included patients' cohorts presented in an abstract form but not published in a peer-reviewed manuscript, which can introduce bias. By limiting the analysis to only multi-fractionated regimens and correcting for publication bias, we believe that the present analysis provides additional valuable information.

We acknowledge several potential limitations of our study. This study represents an analysis of mostly retrospective data with all the usual associated limitations. The results presented also represent findings from a cohort-level study rather than a patient-level meta-analysis. Patients' treatment characteristics were also reported at the study group level. As a result, it was not clear, for example, whether patients with higher risk disease were the patients within a given cohort who received ADT, and whether this impacted associated outcomes. It is also important to note that more than $30 \%$ of the patients re- 
ceived ADT as a part of their definitive treatment course, which may have contributed to the high 5-year bRFS rates. The studies included also did not consistently publish on patient-reported outcomes, which may be discordant with physician-reported outcomes reported in this meta-analysis. Furthermore, most of the included studies focused on reporting of severe toxicities, so we were unable to analyze rates of grade 1-2 toxicities. Rates of erectile dysfunction were also not consistently reported; therefore, this important long-term outcome could not be evaluated in the present analysis.

Our findings demonstrate that primary fractionated HDR brachytherapy as monotherapy is associated with high rates of disease control and low rates of significant toxicity. These results compare favorably to those reported for other modalities, including LDR brachytherapy, hypofractionated external beam radiotherapy, and SBRT. Longer term follow-up can help to distinguish advantages and disadvantages between various treatment options. Future clinical trials would benefit from the inclusion of HDR brachytherapy monotherapy as a treatment arm in order to assess its potential relative benefits compared to other treatment modalities.

\section{Acknowledgements}

We would like to thank Caroline Marshall who assisted with the literature search.

\section{Disclosure}

The authors report no conflict of interest.

The supplemental materials are available on the website of journal.

\section{References}

1. Barnes J, Kennedy WR, Fischer-Valuck BW et al. Treatment patterns of high-dose-rate and low-dose-rate brachytherapy as monotherapy for prostate cancer. J Contemp Brachytherapy 2019; 11: 320-328.

2. Hathout L, Mahmoud O, Wang Y et al. A Phase 2 randomized pilot study comparing high-dose-rate brachytherapy and low-dose-rate brachytherapy as monotherapy in localized prostate cancer. Adv Radiat Oncol 2019; 4: 631-640.

3. Morton G, McGuffin M, Chung HT et al. Prostate high doserate brachytherapy as monotherapy for low and intermediate risk prostate cancer: Efficacy results from a randomized phase II clinical trial of one fraction of $19 \mathrm{~Gy}$ or two fractions of 13.5 Gy. Radiother Oncol 2020; 146: 90-96.

4. Liberati A, Altman DG, Tetzlaff J et al. The PRISMA statement for reporting systematic reviews and meta-analyses of studies that evaluate healthcare interventions: explanation and elaboration. BMJ 2009; 339: b2700.

5. Demanes DJ, Martinez AA, Ghilezan M et al. High-dose-rate monotherapy: safe and effective brachytherapy for patients with localized prostate cancer. Int J Radiat Oncol Biol Phys 2011; 81: 1286-1292.

6. Hauswald H, Kamrava MR, Fallon JM et al. High-dose-rate monotherapy for localized prostate cancer: 10-year results. Int J Radiat Oncol Biol Phys 2016; 94: 667-674.

7. Yoshida K, Yamazaki H, Nakamara S et al. Comparison of common terminology criteria for adverse events v3.0 and ra- diation therapy oncology group toxicity score system after high-dose-rate interstitial brachytherapy as monotherapy for prostate cancer. Anticancer Res 2014; 34: 2015-2018.

8. Yoshioka Y, Kotsuma T, Komiya A et al. Nationwide, multicenter, retrospective study on high-dose-rate brachytherapy as monotherapy for prostate cancer. Int J Radiat Oncol Biol Phys 2017; 97: 952-961.

9. Yamazaki H, Masui K, Suzuki G et al. Comparison of three moderate fractionated schedules employed in high-dose-rate brachytherapy monotherapy for clinically localized prostate cancer. Radiother Oncol 2018; 129: 370-376.

10. Hoskin P, Rojas A, Ostler P et al. Single-dose high-dose-rate brachytherapy compared to two and three fractions for locally advanced prostate cancer. Radiother Oncol 2017; 124: 56-60.

11. Jawad MS, Dilworth JT, Gustafson GS et al. Outcomes associated with 3 treatment schedules of high-dose-rate brachytherapy monotherapy for favorable-risk prostate cancer. Int J Radiat Oncol Biol Phys 2016; 94: 657-666.

12. Patel S, Demanes DJ, Ragab $\mathrm{O}$ et al. High-dose-rate brachytherapy monotherapy without androgen deprivation therapy for intermediate-risk prostate cancer. Brachytherapy 2017; 16: 299-305.

13. Zamboglou N, Tselis N, Baltas D et al. High-dose-rate interstitial brachytherapy as monotherapy for clinically localized prostate cancer: treatment evolution and mature results. Int J Radiat Oncol Biol Phys 2013; 85: 672-678.

14. Roach M, 3rd, Hanks G, Thames H, Jr. et al. Defining biochemical failure following radiotherapy with or without hormonal therapy in men with clinically localized prostate cancer: recommendations of the RTOG-ASTRO Phoenix Consensus Conference. Int J Radiat Oncol Biol Phys 2006; 65: 965-974.

15. Konstantopoulos S. Fixed effects and variance components estimation in three-level meta-analysis. Res Synth Methods 2011; 2: 61-76.

16. Higgins JP, Thompson SG. Quantifying heterogeneity in a meta-analysis. Stat Med 2002; 21: 1539-1558.

17. Egger M, Davey Smith G, Schneider $M$ et al. Bias in meta-analysis detected by a simple, graphical test. BMJ 1997; 315: 629-634.

18. Duval S, Tweedie R. Trim and fill: A simple funnel-plotbased method of testing and adjusting for publication bias in meta-analysis. Biometrics 2000; 56: 455-463.

19. Jackson WC, Silva J, Hartman HE et al. Stereotactic body radiation therapy for localized prostate cancer: a systematic review and meta-analysis of over 6,000 patients treated on prospective studies. Int J Radiat Oncol Biol Phys 2019; 104: 778-789.

20. Frank SJ, Pugh TJ, Blanchard P et al. Prospective phase 2 trial of permanent seed implantation prostate brachytherapy for intermediate-risk localized prostate cancer: efficacy, toxicity, and quality of life outcomes. Int J Radiat Oncol Biol Phys 2018; 100: 374-382.

21. Dearnaley D, Syndikus I, Mossop H et al. Conventional versus hypofractionated high-dose intensity-modulated radiotherapy for prostate cancer: 5-year outcomes of the randomised, non-inferiority, phase $3 \mathrm{CHHiP}$ trial. Lancet Oncol 2016; 17: 1047-1060.

22. Vigneault E, Mbodji K, Magnan S et al. High-dose-rate brachytherapy boost for prostate cancer treatment: Different combinations of hypofractionated regimens and clinical outcomes. Radiother Oncol 2017; 124: 49-55.

23. Martinez AA, Gustafson G, Gonzalez J et al. Dose escalation using conformal high-dose-rate brachytherapy improves outcome in unfavorable prostate cancer. Int J Radiat Oncol Biol Phys 2002; 53: 316-327.

24. Rodda S, Tyldesley S, Morris WJ et al. ASCENDE-RT: an analysis of treatment-related morbidity for a randomized 
trial comparing a low-dose-rate brachytherapy boost with a dose-escalated external beam boost for high- and intermediate-risk prostate cancer. Int J Radiat Oncol Biol Phys 2017; 98: 286-295.

25. Baker BR, Basak R, Mohiuddin JJ et al. Use of stereotactic body radiotherapy for prostate cancer in the United States from 2004 through 2012. Cancer 2016; 122: 2234-2241.

26. Laviana AA, Ilg AM, Veruttipong D et al. Utilizing time-driven activity-based costing to understand the short- and longterm costs of treating localized, low-risk prostate cancer. Cancer 2016; 122: 447-455.

27. Viani GA, Arruda CV, Assis Pellizzon AC et al. HDR brachytherapy as monotherapy for prostate cancer: A systematic review with meta-analysis. Brachytherapy 2021; 20: 307-314. 